\title{
THE TENSOR PRODUCT OF DISTRIBUTIVE LATTICES
}

\author{
by GRANT A. FRASER \\ (Received 17th December 1974)
}

The well-known algebraic concept of tensor product exists for any variety of algebras. The tensor product of groups and of rings have been studied extensively. For other varieties, such as the variety of semigroups, the tensor product has been investigated more recently (5). In this paper we investigate the tensor product of distributive lattices.

We study the tensor product $A \otimes B$ of distributive lattices $A$ and $B$ in the category of distributive lattices. In the first section we give preliminaries on notation. In the second section we prove the existence of the tensor product. We analyse the set of prime filters in $A \otimes B$ and then use Stone's representation theorem for distributive lattices to obtain a method of constructing the tensor product explicitly. In the third section we study the word problem for $A \otimes B$. We show that in general the word problem for $A \otimes B$ is not solvable but we obtain a characterisation for the general case that is the best possible under the circumstances. In the fourth section we restrict our attention to chains. We prove that in this case the word problem has a simple solution and we show that if $A_{1}$ is a sublattice of $A$ and $B_{1}$ a sublattice of $B$ then $A_{1} \otimes B_{1}$ can be embedded as a sublattice of $A \otimes B$. An example is given to show that this assertion is false for arbitrary distributive lattices.

The author would like to thank Alfred Horn for his interest and advice on this work.

\section{Preliminaries}

For terminology and basic results of lattice theory and universal algebra, consult Birkhoff (3) and Grätzer (4) respectively. Let $A$ be a lattice. If $a, b \in A$, the join and meet of $a$ and $b$ are written as $a+b$ and $a b$ respectively. If $a_{i} \in A$ for $i \in I$, where $I$ is any non-empty set, then the join and meet of $\left\{a_{i}: i \in I\right\}$, if they exist, are denoted by $\sum_{i \in I} a_{i}$ and $\prod_{i \in I} a_{i}$ respectively. The smallest and largest elements of $A$, if they exist, are denoted by 0 and 1 respectively. We denote by 2 the two element lattice consisting of 0 and 1 . The category of distributive lattices is denoted by $\mathscr{D}$.

\section{Existence and construction of the tensor product}

Definition 2.1. Let $A, B$ and $C$ be distributive lattices. A function

$$
f: A \times B \rightarrow C
$$


is a bihomomorphism if the functions $g_{a}: B \rightarrow C$ defined by $g_{a}(b)=f(a, b)$ and $h_{b}: A \rightarrow C$ defined by $h_{b}(a)=f(a, b)$ are homomorphisms for each $a \in A$ and $b \in B$.

Definition 2.2. Let $A$ and $B$ be distributive lattices. A distributive lattice $C$ is a tensor product of $A$ and $B$ (in the category $\mathscr{D}$ ) if there exists a canonical bihomomorphism $f: A \times B \rightarrow C$ such that $C$ is generated by $f(A \times B)$ and for any distributive lattice $D$ and any bihomomorphism $g: A \times B \rightarrow D$ there is a homomorphism $h: C \rightarrow D$ satisfying $g=h f$.

Note that since $f(A \times B)$ generates $C$, the homomorphism $h$ is necessarily unique.

Theorem 2.3. Let $A$ and $B$ be distributive lattices. Then a tensor product of $A$ and $B$ in the category $\mathscr{D}$ exists and is unique up to isomorphism.

Proof. Let $K$ be the free distributive lattice on $A \times B$ and let $w$ be the canonical inclusion map of $A \times B$ into $K$. Let $\rho$ be the set of all ordered pairs of the form

$$
\left(w(a, b), w\left(a_{1}, b_{1}\right) * w\left(a_{2}, b_{2}\right)\right) \text {, }
$$

where $a, a_{1}, a_{2} \in A ; b, b_{1}, b_{2} \in B$; * denotes either the join or the meet operation; and either $a=a_{1} * a_{2}$ and $b=b_{1}=b_{2}$, or $a=a_{1}=a_{2}$ and $b=b_{1} * b_{2}$. Let $\sigma$ be the smallest congruence relation in $K$ containing $\rho$. Let $C=K / \sigma, u$ be the canonical homomorphism from $K$ onto $C$, and $f=u w$.

By the choice of $\sigma$, it is clear that $f$ is a bihomomorphism. Let $D$ be any distributive lattice and let $g: A \times B \rightarrow D$ be any bihomomorphism. There is a unique homomorphism $s: K \rightarrow D$ such that $g=s w$. Since $g$ is a bihomomorphism, the kernel relation of $s, \operatorname{ker} s$, contains $\sigma$. Therefore

$$
\text { ker } u=\sigma \subseteq \operatorname{ker} s \text {, }
$$

so that $s=h u$ for some unique homomorphism $h: C \rightarrow D$. Then $h$ is such that $g=s w=h u w=h f$. Finally, since $w(A \times B)$ generates $K, u w(A \times B)$ generates $K / \sigma$, so that $f(A \times B)$ generates $C$. This shows that the distributive lattice $C$ and the canonical bihomomorphism $f$ satisfy the conditions of the definition of a tensor product.

The uniqueness of a tensor product is clear from its definition as a solution of a universal problem.

The tensor product of $A$ and $B$ is denoted by $A \otimes B$ and the image of $(a, b)$ under the canonical bihomomorphism $f: A \times B \rightarrow A \otimes B$ is written as $a \otimes b$. In this notation the proof of Theorem 2.3 shows that $A \otimes B$ is the distributive lattice generated by the elements $a \otimes b(a \in A, b \in B)$, subject to the bihomomorphic conditions

$$
\begin{aligned}
\left(a_{1}+a_{2}\right) \otimes b & =\left(a_{1} \otimes b\right)+\left(a_{2} \otimes b\right), \\
\left(a_{1} a_{2}\right) \otimes b & =\left(a_{1} \otimes b\right)\left(a_{2} \otimes b\right), \\
a \otimes\left(b_{1}+b_{2}\right) & =\left(a \otimes b_{1}\right)+\left(a \otimes b_{2}\right),
\end{aligned}
$$


and

$$
a \otimes\left(b_{1} b_{2}\right)=\left(a \otimes b_{1}\right)\left(a \otimes b_{2}\right)
$$

for all $a, a_{1}, a_{2} \in A$ and $b, b_{1}, b_{2} \in B$. Every element of $A \otimes B$ can be written in the form $\sum_{i=1}^{n} \prod_{j=1}^{k}\left(a_{i j} \otimes b_{i j}\right)$ for some $a_{i j} \in A$ and $b_{i j} \in B, i=1, \ldots, n$, $j=1, \ldots, k$.

We obtain a method of explicitly constructing the tensor product by analysing the set of prime filters in $A \otimes B$.

For a distributive lattice $L$, let $S(L)$ be the set of prime filters in $L$, and let $S^{\prime}(L)=S(L) \cup\{\varnothing, L\}$. It is well-known that there is a one-to-one correspondence between $P \in S^{\prime}(L)$ and homomorphisms $h: L \rightarrow 2$.

It is immediate that there is a one-to-one correspondence between homomorphisms $h: A \otimes B \rightarrow C$ and bihomomorphisms $g: A \times B \rightarrow C$. Thus there is a one-to-one correspondence between $P \in S^{\prime}(A \otimes B)$ and bihomomorphisms $g: A \times B \rightarrow 2$, such that $a \otimes b \in P$ if and only if $g(a, b)=1$. Let $\mathscr{G}$ be the set of all bihomomorphisms $g: A \times B \rightarrow 2$. We order $\mathscr{G}$ by setting $g_{1} \leqq g_{2}$ if and only if $g_{1}(a, b)=1$ implies $g_{2}(a, b)=1$ for all $a \in A, b \in B$. Let

$$
P, Q \in S^{\prime}(A \otimes B)
$$

and let $g_{P}, g_{Q}$ be the corresponding bihomomorphisms. Then

$$
\begin{aligned}
g_{P} \leqq g_{Q} \Leftrightarrow & g_{P}(a, b)=1 \text { implies } g_{Q}(a, b)=1 \\
& \text { for all } a \in A, b \in B \\
\Leftrightarrow & a \otimes b \in P \text { implies } a \otimes b \in Q \\
& \text { for all } a \in A, b \in B \\
\Leftrightarrow & P \subseteq Q .
\end{aligned}
$$

Thus $S^{\prime}(A \otimes B)$ is order isomorphic to $\mathscr{G}$.

Now let $g \in \mathscr{G}$ and for each $x \in A$, let $F(x)=\{y \in B: g(x, y)=1\}$. Then it follows from the bihomomorphic properties of $g$ that $F(x) \in S^{\prime}(B)$ and that for all $x_{1}, x_{2} \in A$,

$$
F\left(x_{1} x_{2}\right)=F\left(x_{1}\right) \cap F\left(x_{2}\right) \text { and } F\left(x_{1}+x_{2}\right)=F\left(x_{1}\right) \cup F\left(x_{2}\right) .
$$

The last equality shows that the union of two members of the range of $F$ is in $S^{\prime}(B)$. But if the union of two filters is a filter, then one must be contained in the other. So the range of $F$ is a chain, and $F$ is a homomorphism from $A$ into a subchain of $S^{\prime}(B)$.

Conversely, if $F$ is such a homomorphism, define $g: A \times B \rightarrow 2$ by setting $g(x, y)=1$ if and only if $y \in F(x)$. It is a straightforward verification that $g$ is a bihomomorphism.

It is also clear from the definitions that if $g_{1}$ and $g_{2}$ are distinct bihomomorphisms, the corresponding homomorphisms $F_{1}$ and $F_{2}$ are also distinct.

Let $\mathscr{H}$ be the set of all homomorphisms from $A$ into a subchain of $S^{\prime}(B)$. 
We order $\mathscr{H}$ by setting $F \leqq G$ if and only if $F(x) \subseteq G(x)$ for all $x \in A$. Let $g_{1}$ and $g_{2}$ be bihomomorphisms and let $F_{1}$ and $F_{2}$ be the corresponding homomorphisms. Then

$$
\begin{aligned}
F_{1} \leqq F_{2} \Leftrightarrow & F_{1}(x) \leqq F_{2}(x) \\
& \text { for all } x \in A \\
\Leftrightarrow & y \in F_{1}(x) \text { implies } y \in F_{2}(x) \\
& \text { for all } x \in A, y \in B \\
\Leftrightarrow & g_{1}(x, y)=1 \text { implies } g_{2}(x, y)=1 \\
& \text { for all } x \in A, y \in B \\
\Leftrightarrow & g_{1} \leqq g_{2} .
\end{aligned}
$$

Thus $\mathscr{H}$ is order isomorphic to $\mathscr{G}$.

We have now proved the following theorem.

Theorem 2.4. Let $A$ and $B$ be distributive lattices. Then $S^{\prime}(A \otimes B)$ is order isomorphic to the set $\mathscr{H}$ of homomorphisms from $A$ into a subchain of $S^{\prime}(B)$.

Let $\mathscr{H}_{0}$ be the set of all $F \in \mathscr{H}$ except the homomorphism $F$ defined by $F(x)=B$ for all $x \in A$.

Corollary 2.5. Let $A$ and $B$ be distributive lattices. Then $A \otimes B$ is isomorphic to the ring of sets generated by the collection $\left\{(a \otimes b)^{*}: a \in A, b \in B\right\}$, where $(a \otimes b)^{*}=\left\{F \in \mathscr{H}_{0}: b \in F(a)\right\}$.

Proof. According to Stone's representation theorem (6), for every distributive lattice $L$, the map $x \rightarrow x^{*}=\{P \in S(L): x \in P\}$ determines an isomorphism between $L$ and a ring of subsets of $S(L)$. In the case of $A \otimes B$, it suffices to consider the restriction of this map to the generators $a \otimes b$ and to determine the ring of sets generated by the sets $(a \otimes b)^{*}$. Now

$$
(a \otimes b)^{*}=\{P \in S(A \otimes B): a \otimes b \in P\} .
$$

From the proof of Theorem 2.4 we have

$$
a \otimes b \in P \Leftrightarrow g(a, b)=1 \Leftrightarrow b \in F(a),
$$

where $P \in S(A \otimes B), g$ is the bihomomorphism corresponding to $P$, and $F$ is the homomorphism corresponding to $g$. In view of the isomorphism between $S^{\prime}(A \otimes B)$ and $\mathscr{H}$ we may write $(a \otimes b)^{*}$ as $\left\{F \in \mathscr{H}_{0}: b \in F(a)\right\}$.

\section{The word problem}

We next study the word problem for the tensor product of distributive lattices. It is enough to consider when a product is less than or equal to a sum. We recall that if $L$ is a distributive lattice and $x, y \in L$, then $x \leqq y$ if and only if for every homomorphism $f: L \rightarrow 2, f(x)=1$ implies $f(y)=1$. 
Lemma 3.1. Let $A$ and $B$ be distributive lattices, and let $a_{i}, c_{j} \in A$ and $b_{i}, d_{j} \in B$ for $i=1, \ldots, n$ and $j=1, \ldots, k$. Then $\prod_{i=1}^{n}\left(a_{i} \otimes b_{i}\right) \leqq \sum_{j=1}^{k}\left(c_{j} \otimes d_{j}\right)$ if and only if for every bihomomorphism $g: A \times B \rightarrow 2, g\left(a_{i}, b_{i}\right)=1$ for all $i$ implies $g\left(c_{j}, d_{j}\right)=1$ for some $j$.

Proof.

$$
\prod_{i=1}^{n}\left(a_{i} \otimes b_{i}\right) \leqq \sum_{j=1}^{k}\left(c_{j} \otimes d_{j}\right)
$$

$\Leftrightarrow$ for every homomorphism $h: A \otimes B \rightarrow 2$,

$$
h\left(\prod_{i}^{n}\left(a_{i} \otimes b_{i}\right)\right)=1 \text { implies } h\left(\sum_{i}^{k}\left(c_{j} \otimes d_{j}\right)\right)=1
$$

$\Leftrightarrow$ for every homomorphism $h: A \otimes B \rightarrow 2$,

$$
h\left(a_{i} \otimes b_{i}\right)=1 \text { for all } i \text { implies } h\left(c_{j} \otimes d_{j}\right)=1 \text { for some } j
$$

$\Leftrightarrow$ for every bihomomorphism $g: A \times B \rightarrow 2$,

$$
g\left(a_{i}, b_{i}\right)=1 \text { for all } i \text { implies } g\left(c_{j}, d_{j}\right)=1 \text { for some } j .
$$

Let $A$ and $B$ be distributive lattices and let $a_{1}, a_{2} \in A$ and $b_{1}, b_{2} \in B$. Suppose that $a_{1} \leqq a_{2}$ and $b_{1} \leqq b_{2}$. Then the order preserving properties of the canonical bihomomorphism imply that $a_{1} \otimes b_{1} \leqq a_{2} \otimes b_{1} \leqq a_{2} \otimes b_{2}$. Thus if $a_{1} \leqq a_{2}$ and $b_{1} \leqq b_{2}$, then $a_{1} \otimes b_{1} \leqq a_{2} \otimes b_{2}$.

For any positive integer $n$, let $n$ be the set $\{1, \ldots, n\}$.

Theorem 3.2. Let $A$ and $B$ be distributive lattices and let $a, a_{i} \in A$ and $b, b_{i} \in B$ for $i=1, \ldots, n$. Then $a \otimes b \leqq \sum_{i=1}^{\text {in }}\left(a_{i} \otimes b_{i}\right)$ if and only if $a \leqq \sum_{i=1}^{n} a_{i}, b \leqq \sum_{i=1}^{n} b_{i}$, and for all $S \subseteq n, a \leqq \sum_{i \in S} a_{i}$ or $b \leqq \sum_{i \notin S} b_{i}$.

Proof. Assume $a \otimes b \leqq \sum_{i=1}^{n}\left(a_{i} \otimes b_{i}\right)$ and suppose that there is a subset $S$ of $n$ such that $a \leqq \sum_{i \in S} a_{i}$ and $b \leqq \sum_{i \notin S} b_{i}$. Then there are homomorphisms $f_{1}: A \rightarrow 2$ and $f_{2}: B \rightarrow 2$ such that $f_{1}(a)=1, f_{1}\left(\sum_{i \in S} a_{i}\right)=0, f_{2}(b)=1$, and

$$
f_{2}\left(\sum_{i \notin S} b_{i}\right)=0 \text {. }
$$

So for each $i \in S, f_{1}\left(a_{i}\right)=0$ and for each $i \notin S, f_{2}\left(b_{i}\right)=0$. Let $g: A \times B \rightarrow 2$ be defined by $g(x, y)=f_{1}(x) f_{2}(y)$. Then $g$ is a bihomomorphism, $g(a, b)=1$ and for all $i \in n, g\left(a_{i}, b_{i}\right)=0$. If we suppose that $a \$ \sum_{i}^{n} a_{i}$, then there is a homomorphism $f: A \rightarrow 2$ such that $f(a)=1$ and $f\left(a_{i}\right)=0$ for all $i \in n$, and we define $g: A \times B \rightarrow 2$ by $g(x, y)=f(x)$. Again $g$ is a bihomomorphism, $g(a, b)=1$ and $g\left(a_{i}, b_{i}\right)=0$ for all $i \in n$. Similarly, if $b \pm \sum_{i}^{n} b_{i}$, there is a bihomomorphism 
with these properties. In each case Lemma 3.1 implies that $a \otimes b \pm \sum_{1}^{n}\left(a_{i} \otimes b_{i}\right)$ and we have a contradiction.

Conversely, suppose that $a \leqq \sum_{1}^{n} a_{i}, b \leqq \sum_{1}^{n} b_{i}$ and for all $S \subseteq n, a \leqq \sum_{i \in S} a_{i}$ or $b \leqq \sum_{i \notin S} b_{i}$. Let $\mathscr{S}=\left\{S \subseteq n: S \neq \varnothing\right.$ and $\left.a \leqq \sum_{i \in S} a_{i}\right\}$ and

$$
\mathscr{T}=\left\{T \subseteq n: T \neq n \text { and } b \leqq \sum_{i \notin T} b_{i}\right\}
$$

Then $\mathscr{S}$ and $\mathscr{T}$ are non-empty since $n \in \mathscr{S}$ and $\varnothing \in \mathscr{T}$. Let $\mathscr{S}=\left\{S_{1}, \ldots, S_{p}\right\}$ and $\mathscr{T}=\left\{T_{1}, \ldots, T_{q}\right\}$. Then

and

$$
a \leqq \prod_{S \in \mathscr{S}} \sum_{i \in S} a_{i}=\sum_{i_{k} \in S_{k}, i \leqq k \leqq p} a_{i_{1}} \ldots a_{i_{p}}
$$

Hence

$$
b \leqq \prod_{T \in \mathscr{T}} \sum_{j \notin T} b_{j}=\sum_{j m \notin T_{m}, 1 \leqq m \leqq q} b_{j_{1}} \ldots b_{j_{q}} \text {. }
$$

$$
\begin{aligned}
a \otimes b & \leqq\left(\sum_{i_{k} \in S_{k}, 1 \leqq k \leqq p} a_{\left.i_{1} \ldots a_{i_{p}}\right) \otimes(} \sum_{j_{m} \notin T_{m}, 1 \leqq m \leqq q} b_{j_{1}} \ldots b_{j_{q}}\right) \\
& =\sum_{i_{k} \in S_{k}, 1 \leqq k \leqq p} \sum_{j_{m} \xi T_{m}, 1 \leqq m \leqq q}\left(a_{i_{1}} \ldots a_{i_{p}} \otimes b_{j_{1}} \ldots b_{j_{q}}\right) .
\end{aligned}
$$

Let $i_{1} \in S_{1}, \ldots, i_{p} \in S_{p}, j_{1} \notin T_{1}, \ldots, j_{q} \notin T_{q}$, and let $J=\left\{j_{1}, \ldots, j_{q}\right\}$. Since $J \subseteq n$, we have $J \in \mathscr{S}$ or $J \in \mathscr{T}$. If $J \in \mathscr{T}$, say $J=T_{r}, r \leqq q$, then $j_{r} \in T_{r}$. But $j_{r} \notin T_{r}$. Hence we must have $J \in \mathscr{S}$. So $J=S_{u}$ for some $u \leqq p$, and $i_{u} \in J$, so $i_{u}=j_{v}$ for some $v \leqq q$. It follows that

$$
\left(a_{i_{1}} \ldots a_{i_{p}}\right) \otimes\left(b_{j_{1}} \ldots b_{j_{q}}\right) \leqq a_{i_{u}} \otimes b_{j_{v}}=a_{i_{u}} \otimes b_{i_{u}} \leqq \sum_{1}^{n}\left(a_{i} \otimes b_{i}\right)
$$

Summing over all terms we obtain

$$
\sum_{i_{k} \in S_{k}, 1 \leqq k \leqq p} \sum_{j_{m} \notin T_{m}, 1 \leqq m \leqq q}\left(a_{i_{1}} \ldots a_{i_{p}} \otimes b_{j_{1}} \ldots b_{j_{q}}\right) \leqq \sum_{1}^{n}\left(a_{i} \otimes b_{i}\right)
$$

and hence $a \otimes b \leqq \sum_{1}^{n}\left(a_{i} \otimes b_{i}\right)$.

By dualising Theorem 3.2 we obtain a characterisation of an inequality of the form $\prod_{1}^{n}\left(a_{i} \otimes b_{i}\right) \leqq a \otimes b$. However, it is not possible to characterise a more general inequality solely in terms of the relations among the elements of $A$ and $B$ involved in the inequality. Specifically, an inequality of the form $\prod_{i=1}^{n}\left(a_{i} \otimes b_{i}\right) \leqq \sum_{j=1}^{k}\left(c_{j} \otimes d_{j}\right)$ cannot be so characterised.

As an example, let $A=2, B_{1}=3$ (the three-element chain $\{0, a, 1\}$ satisfying $0<a<1$ ), and $B_{2}=$ the diamond (the four-element lattice $\{0, a, b, 1\}$ with $a$ and $b$ incomparable). Then the inequality $(0 \otimes 1)(1 \otimes a) \leqq(0 \otimes a)+(1 \otimes 0)$ fails in $A \otimes B_{1}$ and holds in $A \otimes B_{2}$. This can be shown using Lemma 3.1. To prove the first assertion, we must construct a bihomomorphism $g: A \times B_{1} \rightarrow 2$ 
such that $g(0,1)=g(1, a)=1$ and $g(0, a)=g(1,0)=0$. We define $g$ as above and we set $g(0,0)=0$ and $g(1,1)=1$; then it is clear that the function $g$ is a bihomomorphism. To prove the second assertion, we must show that there is no bihomomorphism $g: A \times B_{2} \rightarrow 2$ such that $g(0,1)=g(1, a)=1$ and $g(0, a)=g(1,0)=0$. Suppose $g$ is such a bihomomorphism. Then $1=g(0,1)=g(0, a)+g(0, b)=g(0, b) \leqq g(1, b)$

and we have a contradiction.

$$
=g(1, b) g(1, a)=g(1,0)=0
$$

This example shows that, in general, an inequality in a tensor product $A \otimes B$ depends not only on the relations among the elements of $A$ and $B$ involved in the inequality, but also on the lattices $A$ and $B$ themselves.

Let $A_{1}$ be a sublattice of $A$ and $B_{1}$ a sublattice of $B$. We consider the problem of whether $A_{1} \otimes B_{1}$ can be embedded as a sublattice of $A \otimes B$. Let $\otimes_{1}$ be the canonical bihomomorphism from $A_{1} \times B_{1}$ into $A_{1} \otimes B_{1}$ and let $\otimes$ be the restriction to $A_{1} \times B_{1}$ of the canonical bihomomorphism from $A \times B$ into $A \otimes B$. It follows from the definition of the tensor product that there is a canonical homomorphism $h$ from $A_{1} \otimes B_{1}$ into $A \otimes B$ such that for all $a \in A_{1}$ and $b \in B_{1}, h\left(a \otimes_{1} b\right)=a \otimes b$. If $h$ is one-to-one, $A_{1} \otimes B_{1}$ can be canonically embedded as a sublattice of $A \otimes B$ and we say that $A_{1} \otimes B_{1}$ is canonically isomorphic to a sublattice of $A \otimes B$.

The example given above can be used to settle this problem in the negative. In the example, $A$ is a sublattice of itself and $B_{1}$ is a sublattice of $B_{2}$. If $A \otimes B_{1}$ is canonically isomorphic to a sublattice of $A \otimes B_{2}$, then an inequality holds in $A \otimes B_{1}$ if and only if its image under the canonical homomorphism holds in $A \otimes B_{2}$. But the inequality given in the example fails in $A \otimes B_{1}$ and holds in $A \otimes B_{2}$.

We shall show in Section 4 that this problem has an affirmative solution when $A$ and $B$ are chains.

The following result characterises the join-irreducible generators of the tensor product.

Theorem 3.3. Let $A$ and $B$ be distributive lattices and let $a \in A$ and $b \in B$. Then $a \otimes b$ is join-irreducible in $A \otimes B$ if and only if $a$ is join-irreducible in $A$ and $b$ is join-irreducible in $B$.

Proof. Let $a \otimes b$ be join-irreducible and suppose $a \leqq \sum_{i=1}^{n} a_{i}$. Then

$$
a \otimes b \leqq\left(\sum_{1}^{n} a_{i}\right) \otimes b=\sum_{1}^{n}\left(a_{i} \otimes b\right) .
$$

Hence $a \otimes b \leqq a_{i} \otimes b$ for some $i$ and so $a \leqq a_{i}$. Thus $a$ is join-irreducible. Similarly $b$ is join-irreducible.

Now suppose $a$ and $b$ are join-irreducible; we may assume

$$
a \otimes b \leqq \sum_{i=1}^{n}\left(a_{i} \otimes b_{i}\right)
$$


and we use Theorem 3.2. Let $S=\left\{j \in n: b \leqq b_{j}\right\}$. If $S=n$ then $a \leqq \sum_{j \in S} a_{j}$. If $S \neq n$ we again have $a \leqq \sum_{j \in S} a_{j}$, for otherwise $b \leqq \sum_{j \notin S} b_{j}$ and hence $b \leqq b_{j}$ for some $j \notin S$. Now $S \neq \varnothing$, since $b \leqq \sum_{j=1}^{n} b_{j}$ and so $b \leqq b_{j}$ for some $j \in n$. Hence $a \leqq \sum_{j \in S} a_{j}$ implies that $a \leqq a_{j}$ for some $j \in S$, and since $b \leqq b_{j}$ we have $a \otimes b \leqq a_{j} \otimes b_{j}$. Thus $a \otimes b$ is join-irreducible.

The generalisation of Theorem 3.3, that $\prod_{i=1}^{n}\left(a_{i} \otimes b_{i}\right)$ is join-irreducible if and only if $a_{1}, \ldots, a_{n}$ and $b_{1}, \ldots, b_{n}$ are join-irreducible, is false for arbitrary distributive lattices $A$ and $B$. For example, if $A$ and $B$ are the diamond $\{0, a, b, 1\}$, then $a$ and $b$ are join-irreducible, but $(a \otimes a)(b \otimes b)$ is not joinirreducible in $A \otimes B$. In fact, $(a \otimes a)(b \otimes b)=(a \otimes 0)(0 \otimes b)+(0 \otimes a)(b \otimes 0)$. This equality can be established with the aid of Lemma 3.1. To see that the righthand side is less than or equal to the left-hand side, note that if $g: A \times B \rightarrow 2$ is any bihomomorphism such that either

$$
g(a, 0)=g(0, b)=1 \text { or } g(0, a)=g(b, 0)=1,
$$

then $g(a, a)=g(b, b)=1$. The reverse inequality holds because there is no bihomomorphism $g: A \times B \rightarrow 2$ such that $g(a, a)=g(b, b)=1, g(a, 0) g(0, b)=0$, and $g(0, a) g(b, 0)=0$. (If there were such a bihomomorphism $g$, say

then

$$
g(a, 0)=g(b, 0)=0,
$$

$$
0=g(a, 0)+g(b, 0)=g(1,0)=g(1, a) g(1, b) \geqq g(a, a) g(b, b)=1 .
$$

The other cases are similar.)

In the next section an extension of Theorem 3.3 gives a characterisation of the join-irreducible elements of $A \otimes B$, when $A$ and $B$ are chains.

\section{The tensor product of chains}

We now study $A \otimes B$ when $A$ and $B$ are chains. We obtain a simple description of the join-irreducible elements of $A \otimes B$ and we show that the word problem for $A \otimes B$ has an elementary solution.

We first obtain a sharpened version of Theorem 3.2 when $A$ and $B$ are chains.

Theorem 4.1. Let $A$ and $B$ be chains and let $a, a_{i} \in A$ and $b, b_{i} \in B$ for $i=1, \ldots, n$. Then $a \otimes b \leqq \sum_{i=1}^{n}\left(a_{i} \otimes b_{i}\right)$ if and only if $a \leqq a_{i}$ and $b \leqq b_{i}$ for some $i$.

Proof. If $a \leqq a_{i}$ and $b \leqq b_{i}$ for some $i$, then $a \otimes b \leqq a_{i} \otimes b_{i} \leqq \sum_{1}^{n}\left(a_{i} \otimes b_{i}\right)$. Conversely, suppose $a \otimes b \leqq \sum_{1}^{n}\left(a_{i} \otimes b_{i}\right)$. By Theorem 3.3, $a \otimes b$ is joinirreducible in $A \otimes B$, so $a \otimes b \leqq a_{i} \otimes b_{i}$ for some $i$, and hence $a \leqq a_{i}$ and $b \leqq b_{i}$. 
Using Theorem 4.1 we characterise the join-irreducible elements in the tensor product of chains.

Theorem 4.2. Let $A$ and $B$ be chains. The join-irreducible elements of $A \otimes B$ are all the products of generators $\prod_{i=1}^{n}\left(a_{i} \otimes b_{i}\right)$, where $a_{i} \in A$ and $b_{i} \in B$ for $i=1, \ldots, n$.

Proof. Clearly any join-irreducible element of $A \otimes B$ must be of the form $\prod_{1}^{n}\left(a_{i} \otimes b_{i}\right)$. Now suppose $\prod_{1}^{n}\left(a_{i} \otimes b_{i}\right)$ is any product of generators of $A \otimes B$. It is enough to show that for any $c_{1}, \ldots, c_{k} \in A$ and $d_{1}, \ldots, d_{k} \in B$,

$$
\prod_{i=1}^{n}\left(a_{i} \otimes b_{i}\right) \leqq \sum_{j=1}^{k}\left(c_{j} \otimes d_{j}\right)
$$

implies that $\prod_{1}^{n}\left(a_{i} \otimes b_{i}\right) \leqq c_{j} \otimes d_{j}$ for some $j$. So assume that

$$
\prod_{1}^{n}\left(a_{i} \otimes b_{i}\right) \leqq \sum_{1}^{k}\left(c_{j} \otimes d_{j}\right)
$$

and suppose $\prod_{1}^{n}\left(a_{i} \otimes b_{i}\right) \leq c_{j} \otimes d_{j}$ for all $j . \quad$ It follows from the dual version of Theorem 4.1 that for each $j, a_{i} \leq c_{j}$ or $b_{i} d_{j}$ for all $i$. Let $g: A \times B \rightarrow 2$ be defined by $g(x, y)=1$ if and only if $x \geqq a_{i}$ and $y \geqq b_{i}$ for some $i$. It is a straightforward verification that $g$ is a bihomomorphism and it is clear that $g\left(a_{i}, b_{i}\right)=1$ for all $i$. Also for all $i$ and $j, c_{j}<a_{i}$ or $d_{j}<b_{i}$, so $g\left(c_{j}, d_{j}\right)=0$ for all $j$. Then it follows from Lemma 3.1 that $\prod_{1}^{n}\left(a_{i} \otimes b_{i}\right) \equiv \sum_{1}^{k}\left(c_{j} \otimes d_{j}\right)$ and we have a contradiction.

Theorem 4.3. Let $A$ and $B$ be chains and let $a_{i}, c_{j} \in A$ and $b_{i}, d_{j} \in B$ for $i=1, \ldots, n$ and $j=1, \ldots, k$. Then $\prod_{i=1}^{n}\left(a_{i} \otimes b_{i}\right) \leqq \sum_{j=1}^{k}\left(c_{j} \otimes d_{j}\right)$ if and only if $a_{i} \leqq c_{j}$ and $b_{i} \leqq d_{j}$ for some $i$ and $j$.

Proof. We first note that the dual version of Theorem 3.3 implies that every generator of $A \otimes B$ is meet-irreducible. Then it follows from Theorem 4.2 and this remark that

$$
\begin{aligned}
\prod_{i=1}^{n}\left(a_{i} \otimes b_{i}\right) \leqq \sum_{j=1}^{k}\left(c_{j} \otimes d_{j}\right) & \Leftrightarrow \prod_{i=1}^{n}\left(a_{i} \otimes b_{i}\right) \leqq c_{j} \otimes d_{j} \text { for some } j \\
& \Leftrightarrow a_{i} \otimes b_{i} \leqq c_{j} \otimes d_{j} \text { for some } i \text { and } j \\
& \Leftrightarrow a_{i} \leqq c_{j} \text { and } b_{i} \leqq d_{j} \text { for some } i \text { and } j
\end{aligned}
$$

Theorem 4.3 shows that the word problem for the tensor product of chains has a simple solution.

Theorem 4.4. Let $A$ and $B$ be chains and let $A_{1}$ be a sublattice of $A$ and $B_{1}$ be a sublattice of $B$. Then $A_{1} \otimes B_{1}$ is canonically isomorphic to a sublattice of $A \otimes B$.

$$
\text { E.M.S.-20/2-1 }
$$


Proof. We recall from the discussion of this problem in Section 3 that the canonical homomorphism $h: A_{1} \otimes B_{1} \rightarrow A \otimes B$ is defined by $h\left(a \otimes_{1} b\right)=a \otimes b$, where $a \in A_{1}, b \in B_{1}$ and $\otimes_{1}$ and $\otimes$ are the canonical bihomomorphisms from $A_{1} \times B_{1}$ to $A_{1} \otimes B_{1}$ and from $A \times B$ to $A \otimes B$ respectively. We need to show that $h$ is one-to-one. Let $\sum_{i} \prod_{j}\left(a_{i j} \otimes_{1} b_{i j}\right)$ and $\sum_{m} \prod_{n}\left(c_{m n} \otimes_{1} d_{m n}\right)$ be distinct elements of $A_{1} \otimes B_{1}$, say

Then by Theorem 4.3,

$$
\sum_{i} \prod_{j}\left(a_{i j} \otimes_{1} b_{i j}\right) \pm \sum_{m} \prod_{n}\left(c_{m n} \otimes_{1} d_{m n}\right)
$$

$$
\sum_{i} \prod_{j}\left(a_{i j} \otimes b_{i j}\right) \$ \sum_{m} \prod_{n}\left(c_{m n} \otimes d_{m n}\right) \quad \text { in } A \otimes B .
$$

Hence

$$
h\left(\sum_{i} \prod_{j}\left(a_{i j} \otimes_{1} b_{i j}\right)\right) \leqq h\left(\sum_{m} \prod_{n}\left(c_{m n} \otimes_{1} d_{m n}\right)\right)
$$

and so the canonical homomorphism $h$ is an isomorphism.

We conclude by showing that the tensor product of $\mathscr{D}$-projective chains is $\mathscr{D}$-projective.

Theorem 4.5. Let $A$ and $B$ be $\mathscr{D}$-projective chains. Then $A \otimes B$ is $\mathscr{D}$ projective.

Proof. Suppose $A$ and $B$ are $\mathscr{D}$-projective chains. By (1, Theorem 8.2), a chain is $\mathscr{D}$-projective if and only if it is countable. Hence $A$ and $B$ are countable, so $A \otimes B$ is also countable. By (2, Theorem 9), a countable distributive lattice is $\mathscr{D}$-projective if and only if every element is a finite sum of join-irreducible elements, every element is a finite product of meet-irreducible elements, and the product of any two join-irreducible elements is joinirreducible.

Now Theorem 4.2 asserts that the join-irreducible elements of $A \otimes B$ are of the form $\prod_{1}^{n}\left(a_{i} \otimes b_{i}\right)$, and the dual version of Theorem 4.2 shows that the meet-irreducible elements are of the form $\sum_{1}^{k}\left(c_{j} \otimes d_{j}\right)$. Since every element of the tensor product can be written as $\sum_{i=1}^{p} \prod_{j=1}^{q}\left(x_{i j} \otimes y_{i j}\right)$ and also as

$$
\prod_{m=1}^{r} \sum_{n=1}^{s}\left(u_{m n} \otimes v_{m n}\right)
$$

it is clear that every element is a finite sum of join-irreducible elements and a finite product of meet-irreducible elements. The product of two join-irreducible elements is again of the form $\prod_{1}^{n}\left(a_{i} \otimes b_{i}\right)$, and so is join-irreducible. Hence $A \otimes B$ satisfies the above conditions and so is $\mathscr{D}$-projective. 
The tensor product of $\mathscr{D}$-projective distributive lattices is not necessarily $\mathscr{D}$-projective. For example, let $A$ and $B$ be the diamond $\{0, a, b, 1\}$. Then $A$ and $B$ are $\mathscr{D}$-projective. By Theorem 3.3, $a \otimes a$ and $b \otimes b$ are join-irreducible in $A \otimes B$, and we saw at the end of Section 3 that $(a \otimes a)(b \otimes b)$ is not joinirreducible in $A \otimes B$. But by (1, Theorem 7.1), a finite distributive lattice is $\mathscr{D}$-projective if and only if the product of any two join-irreducible elements is join-irreducible. Hence $A \otimes B$ is not $\mathscr{D}$-projective.

\section{REFERENCES}

(1) R. Balbes, Projective and injective distributive lattices, Pacific J. Math. 21 (1967), 405-420.

(2) R. Balbes and A. HoRn, Projective distributive lattices, Pacific J. Math. 33 (1970), 273-279.

(3) G. Birkhoff, Lattice theory, 3rd ed. (Colloq. Publ., Vol. 25, Amer. Math. Soc., Providence, R.I., 1967).

(4) G. GRÄTZER, Universal algebra (Van Nostrand, Princeton, N.J., 1968).

(5) P. A. Grillet, The tensor product of semigroups, Trans. Amer. Math. Soc. 138 (1969), 267-280.

(6) M. H. STONE, Topological representations of distributive lattices and Brouwerian logics, Căsopis Pěst. Mat. Fys. 67 (1937), 1-25.

University of Santa Clara

Santa Clara, California 95053 